\title{
Magnetic Resonance Imaging of the Pelvis with and without Contrast
}

National Cancer Institute

\section{Source}

National Cancer Institute. Magnetic Resonance Imaging of the Pelvis with and without

Contrast. NCI Thesaurus. Code C137933.

Magnetic resonance imaging of the pelvis with and without the use of a contrast agent to enhance the image. 